\title{
Designing a Framework to Control The Spread of COVID-19 by Utilizing Cellular System
}

\author{
Awder Mohammed Ahmed \\ Communication Engineering Department \\ Technical College of Engineering \\ Sulaimani Polytechnic University \\ Sulaimani, Iraq \\ awder.ahmed@spu.edu.iq
}

\begin{tabular}{l} 
Article Info \\
\hline Special Issue on \\
Coronavirus (COVID-19) \\
DOI: \\
10.24017/covid.16 \\
Article history: \\
Received 25 May 2020 \\
Accepted 30 May 2020 \\
\hline
\end{tabular}

Keywords:

COVID-19,

Cellular System,

Cell Tower,

Mobile Phone,

Haversine Formula.

\begin{abstract}
Nowadays coronavirus (COVID--19) has become a concerning issue in the world, according to WHO latest report more than 8,000000 of people in our planet have been infected and more than 400000 of people have died until the writing of this paper. Everybody on this planet is exposed to get this pandemic. Scientists, organizations, governments, and universities around the world endeavor to find proper solutions to control the spread of COVID-19. Thus, the aim of this paper is to propose an applicable framework to control the spread of COVID-19 by utilizing Cellular System. This is done by Haversine formula to calculate the distance between mobile phones based on cell towers. And it can also be fulfilled via designing a framework which is composed of four phases. Phase 1, deals with distance calculation and data collection about the infected and suspected persons. Then in phase 2, the Authorized Clinical Center (ACC) delivers the ID of the infected person to the Telecom Company (TC) to gather information about the unknown suspected persons. In turn, phase 3 diagnoses the infected persons and informs them to visit the ACC. In the last phase, the suspected persons are tested and the infected ones are recognize.
\end{abstract} All rights reserved.

\section{INTRODUCTION}

Nowadays Coronavirus pandemic named COVID-19 has become a global health problem on this planet, which till recent date more than 8 million of confirmed cases have been registered and more than 400000 of people have died. 
The physical structure of communities, such as vicinity to assets like basic need stores, green space, the blend of businesses, conveniences, and lodging, influences the COVID-19 widespread [1] meaning that, it is revealed that on one hand, COVID-19 has a direct impact on different sectors such as; transportation, agriculture, and businesses and on the other hand, it leaves a negative impact on our health and humanity of this planet. The virus originated in Wuhan, China, where in December 2019 a cluster of 27 cases of pneumonia of unknown etiology was observed [2]. According to [3], WHO report more than 215 nations, regions and regions have detailed cases of the infection to date and it's widespread has overpowered some of the foremost progressed and sophisticated wellbeing frameworks within the world. From the very beginning of coronavirus spreading, for the control and prevention of spread of COVID-19 in some countries, governments have practiced quarantine process, but the fact is to what extend this process will be continuing. A huge number of employees in private sectors have lost their jobs. Thus, the world has faced a deep recession and so the global economy might go into recession in the near future. Epidemiologically, the morbidity and mortality rate has dramatically increased due to coronavirus [4]. COVID-19 has a brooding period that shifts from 2 days to 14 days and is initially characterized by flu like side effects but may lead into creating serious hacks, shortness of breath, stomach pain, loose bowels, sore throat, misfortune of taste and scent, tiredness, muscle aches/pain, rehashed shaking with chills, and fever[1]. COVID-19 is one of the seven sorts of coronaviruses that taints people, regularly driving to genuine upper respiratory issues which cause illness [1]. As mentioned earlier and from the above quotes, it is revealed that COVID-19 will be represented as one of the most affective types of pandemic disease that has been known to human being. The COVID-19 has put humanity in front of a big challenge. Furthermore, it is the responsibility of Governments, organizations, and universities to take control of the spreading of this pandemic disease. Thus from the beginning of coronavirus spreading, the infected countries have started to perform different techniques and procedures to control the spread of the virus.

From this perspective, this paper aims to propose an applicable framework to control the spread of COVID-19 by utilizing Cellular System in which the facilities that are provided by this technology will be used as a key factor to implement the proposed Framework properly. By dividing the Framework into 4 phases, where in phase 1, the information of the infected and suspected persons is sent to the main office (a specific telecom company) via the Cell towers and then the data is stored. In phase 2, the Authorized Clinical Center sends the infected person's ID to the telecom company. In turn, in phase 3, the Telecom Company determines the people who were around the infected person and treating them as suspected persons and sends their data to the Authorized Clinical Center. Then, in phase 4, the Clinical Center subjects the suspected persons to take medical examinations and this is done in private where people's privacy is taken into consideration. And finally the new infected people are sent to be quarantined and to get the sufficient treatment and those with negative results are let free.

\section{RELATED WORK}

Over recent years, Cellular System or mobile technology has become a part of our daily routine activities; a huge number of activities such as transactions, transportations, marketing will be achieved by utilizing this technology. Furthermore, this technology has been used widely to facilitate the management process of different sectors such in healthcare system. There are limited numbers of researches that have been published till now regarding the addressing of the COVID-19 through the use of Information technology and cellular system because this Virus has started to spread with the start of 2020. Moreover, most of the Governments, researchers, and organizations have focused on finding a reliable COVID-19 vaccine. In whole, there are numbers of researches that have been developed to detect and address the COVID-19 virus. According to [5] a deep learning technique based on Convolution Neural Network (CNN) has been implemented to disclose COVID-19 disease by utilizing Chest X-ray images. In addition, an architecture called modified AlexNet has been 
proposed which compares the two datasets and uses convolutional neural network and this implies that architectures have a significant ability of reveal COVID-19 from X-ray. In [6] the authors proposed both of Artificial Intelligence (AI) and Big data as suitable technology to prevent the spread of COVID-19 and the severe effects of the COVID-19 pandemic. Where this paper shows that, Reverse Transcription Polymerase Chain Reaction (RT-PCR) test is a standard technique to combat the COVID-19 pandemic, but this standard might not be suitable to be utilized by every country because on one hand, some countries have limited budgets and cannot get enough testing kits. And on the other hand, it is time-consuming. They proposed as an alternative combining AI framework with smart device and cloud computing to tackle the issue. And the role of Big data have been raised as Big data relate with cleverly AI-based tools that can construct complex recreation models utilizing coronavirus data streams for episode estimation. And finally, different applications of Big data related to address COVID-19 pandemic have been presented.

From the related works that have been presented in this section, it is revealed that technology has a great impact to address COVID-19 pandemic. Thus, we aim to take this opportunity to control the spread of pandemic by utilizing Cellular System through designing applicable Framework to disclose the suspected people with COVID-19 pandemic while having switched on mobile phone.

\section{CELL TOWER}

Nowadays, the numbers of mobile phone users have rapidly increased especially in urban areas because through this technology people can perform their daily activities easily. At the same time, in order to serve all of those mobile phones with a good quality of signal, telecom companies need to increase the number of cell towers to cover the required areas because the cell towers will be represented as a unique point to connect mobile phones to together. In over the world the number of cell phones and cell towers are dramatically increasing and the conspicuous developments in cell phone technology have made our life much easier [7]. The individual cell phone is controlled by communicating with installation of cell tower (base station) or a telecommunications structure [8]. In other words, it is revealed that mobile phones are getting and transmitting its services via cell towers and the processes of phone calling, messaging, and internet browsing are accomplished by the closer cell tower from our cell phone which depends on signal strength. Furthermore, each cell phone is surrounded by a number of cell towers while moving, but this device will transmit the data via one cell tower at the same time. The geographical distribution and signal strength of the samples in the same BS will determine the location and type of the base station [9]. Cell phone communicates in the range of radio frequency, which is a low frequency non-ionizing radiation. A mobile phone base station is designed in such a way that the cell phones coming under its coverage area should be able to transmit and receive enough signals which enable proper communication within a few kilometers [7]. Each mobile network (Telecom Company) seeks to have clear information about the number of mobile phones that are connected on each cell tower, to reach that, the procedure of connection between the base station and mobile phone is accomplished through; First, the mobile phone network should detect the mobile phone users around the covered areas, this is to determine who and how many users are there. Second, when mobile phones send data to the cell tower, it chops up time into some modicum and then the Cell tower allocates each of those tiny time slots to a specific mobile phone. Third, the procedure of allocating different time slot should be accomplished at this stage.

Let's consider this scenario; Consider if there are 3 mobile phones and they need to allocate time in order to connect with the cell tower. When those phones arrived at the base station while moving they have to arrange when they have arrived based on the allocated time slot by cell tower with different distances. As a result correct time slots will be assigned to each mobile phone. Fourth, in order to improve the communication between base station and mobile phone, it does not have to bide for it is slot time instead the distance between mobile phone and cell tower should be calculated. 
GSM technique is used by telecom companies to observe and detect the number of mobile phones which are connected with a band of particular cell tower, furthermore, the distance between cell tower and mobile phones while moving is calculating by this protocol. There are a number of approaches that ask for RSS to be transformed into distance. Radio propagation models are used to reach such transformations. Naturally, Network Measurements Report (NMR) is sent by any GSM phone at a rate of $480 \mathrm{~ms}$. And thus, the location of any active subscriber is easily calculated by the core network [10]. In general, multiple antennas which are called sectors have been plugged on a Cell tower which provide signal in limited area to mobile phones. The number of supported mobile phones depends on sector type or model. For Mobile Station and Cell tower, radio link specific merits such as; TOA (Time Of Arrival), RSS (Received Signal Strength), and PDP (Power-Delay Profile), are extracted from obtained signals and then are utilized by a special algorithm to calculate an estimate of the Mobile station location [11]. The use of each of those techniques depends on different factors. In other words, for instance; the accuracy outcome form the use of those techniques depends on environment of the place. According to [12] there are two types of localization techniques that are existed; geometrical and non- geometrical. In the first type, the related equations will be assigned between the known positions of the cell tower with unknown position of the mobile station and the calculation of the mobile position. While, the second type utilizes the fingerprinting method and cooperative mobiles in order to calculate mobile position.

\section{DISTANCE MEASUREMENT}

This section aims to find a proper technique in order to find the distance between the infected people by covi-19 and suspected persons through their mobile phones. And then subjects the suspected persons to take a coronavirus test, thus the concerned authorities can easily isolate the infected people and direct them to quarantine.

Through the search there are different equations and formulas have been found in order to calculate the required distance. As a result the Haversine formula is proposed in this paper to be used as a proper formula for distance calculation. According to [13], in order to find a straight distant line between two coordinates on the earth using longitude and latitude parameters the Haversine Formula is an essential equation, this algorithm discusses the shapes of sides and angles in spherical triangles. The reliable academic authority revealed that in 1805, a scientist created a Haversine table to determine a distance between points and the Haversine equation is utilized to calculate the space between user's area and the point of planning destination [14]. The distance calculation between 2 or more mobile users is the key point that will be used as the core issue in this proposed framework in which the distance should be in meter. Additionally, Haversine formula is represented as great circle space (removal), this equation performs calculation from main point to the goal point with trigonometric work by using scope and longitude which produces distance data between two points in meters [15]. Generally, (Mobile Station) MS location is composed of two or three dimensions and it may include information such as; the latitude and longitude of where the MS is located [14]. In order to calculate the distance between two points, the Haversine formula is the proper formula in this regard, which latitude is represented as input and longitude as the initial and last point.

\subsection{Implementation of Haversine Formula}

For the purpose of calculating the distance between two points, the Haversine formula is utilized, in which each of $\theta_{1}$ and $\theta_{2}$ have been represented as Latitude of Mobill and Mobile2, and $\lambda 1$ and $\lambda 2$ as Longitude of Mobill and Mobile2. The output is the value of the distance between the locations of the two mobile phones.

Legend:

$\mathrm{d}$ : distance between the two points.

$\theta_{1}, \theta_{2}$ : Latitude of first point and Latitude of Second point (Latitude of Mobil1 and Mobile2). 
$\lambda 1, \lambda 2$ : Longitude of first point and Latitude of Second point (Longitude of Mobil1 and Mobile2).

r: earth's radius $(6371 \mathrm{~km})$.

$\sin ^{-1}\left(\sqrt{\sin ^{2}\left(\frac{\theta_{2}-\theta_{1}}{2}\right)+\cos \theta_{1} * \cos \theta_{2} * \sin ^{2}\left(\frac{\lambda_{2}-\lambda_{1}}{2}\right)}\right)$

\subsection{Experimental Results}

This sub-section is dedicated to calculate the distances between the mobile phones' position through the Haversine Formula, to get accurate results; the calculation test has been implemented on 10 proposed cell towers. The calculation has been done by utilizing MATLAB-R2020a as a computer programming language as follow:

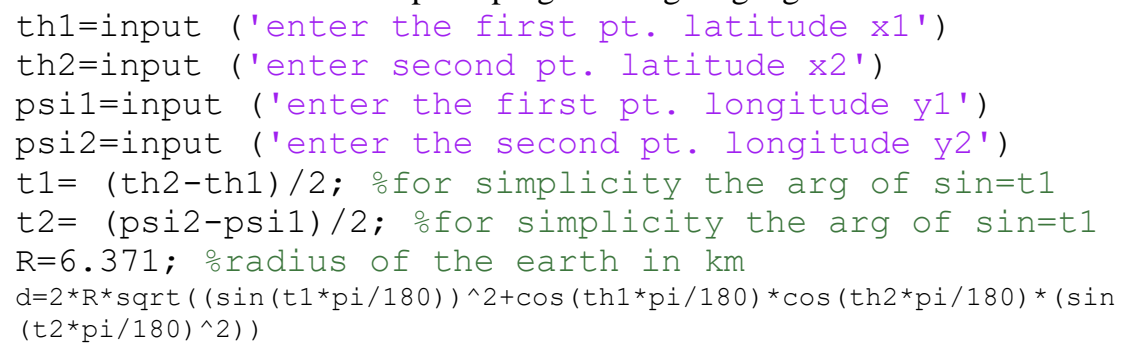

Table 1: Distance Calculations of Mobile Phones

\begin{tabular}{ccccccc}
\hline No: & Location & $\begin{array}{c}\text { Latitude of } \\
\text { Mobile 1 }\end{array}$ & $\begin{array}{c}\text { Longitude of } \\
\text { Mobile 1 }\end{array}$ & $\begin{array}{c}\text { Latitude of } \\
\text { Mobile 2 }\end{array}$ & $\begin{array}{c}\text { Longitude of } \\
\text { Mobile 2 }\end{array}$ & Distance \\
\hline $\mathbf{1}$ & Cell Tower A & 1 & 2 & 2 & 2 & 0.1572 \\
\hline $\mathbf{2}$ & Cell Tower B & 1.5 & 2 & 2 & 1.80 & 0.0599 \\
\hline $\mathbf{3}$ & Cell Tower C & 1.80 & 2 & 2.1 & 2 & 0.0334 \\
\hline $\mathbf{4}$ & Cell Tower D & 2 & 2.1 & 1.6 & 1.5 & 0.0802 \\
\hline $\mathbf{5}$ & Cell Tower E & 2.1 & 2.5 & 3 & 1.3 & 0.1667 \\
\hline $\mathbf{6}$ & Cell Tower F & 3 & 1.8 & 2 & 2 & 0.0222 \\
\hline $\mathbf{7}$ & Cell Tower G & 3.2 & 1.7 & 2.8 & 1.7 & 0.0445 \\
\hline $\mathbf{8}$ & Cell Tower H & 2.6 & 1.6 & 3 & 1.4 & 0.0497 \\
\hline $\mathbf{9}$ & Cell Tower I & 2.8 & 1.5 & 2.3 & 1.9 & 0.0712 \\
\hline $\mathbf{1 0}$ & Cell Tower J & 3.6 & 1 & 3 & 1.2 & 0.0703 \\
\hline
\end{tabular}

Table 1 shows the results of calculated distances between mobile phones. Where, Cell Tower A-J represents the number of Cell Towers at 10 different locations, while Latitude of Mobile 1 and 2 and Longitude of Mobile 1 and 2 represent the Latitude location of Mobile 1 and 2 and Longitude location of Mobile1 and 2 respectively. Based on the obtained results as expressed on equation (1), we propose this procedure to be implemented by Telecom companies to calculate the distances between mobile phones' subscribers. This calculation procedure will be issued in the first phase of the proposed framework, by implementing this procedure the socalled telecom company is able to diagnose the distances between subscribers via mobile phones and Cell towers. Furthermore, the data of the calculated distance can be kept there and based on request by an authorized Clinical Center; the Telecom company in turn can retrieve 
those data to designate the subscribes who were around the infected person (subscriber Id) during the last 14 days.

\section{PROPOSED FRAMEWORK}

In this section, we present a proposed framework to collect the data about the infected and suspected persons with COVID-19 based on the mobile phones distances that have been calculated in section 4 (distance measurement) and then inform the suspected people to take the COVID-19 test and get the required instructions and treatments by an authorized clinical center of the hospital. By implementing this framework properly, the healthcare authority can collect data about suspected people with COVID-19 easily and perform the required procedures. For the implementation of the proposed work, this framework has been categorized in to 4 phases as follow:

1- Phase One (Cell Tower):

In section 3 , it has been clarified that mobile phone transmits its services such as phone call, messaging, and Internet browsing through the nearest cell tower based of signal strength. Each cell tower cover a limited signal area and all switched on mobile phones with the same radio frequency are in a direct contact with this cell tower. In turn, the cell tower is transmitting the mobile phones data into the Mobile central Office and those data will be kept for a period of time for different purposes. As a result, the so-called telecom company has the number and ID of mobile phones that have been covered by a certain cell tower concurrently. In section 4 , the distance calculation between the mobile phones of subscribers has been done. Thus, from this point of view this paper proposes the procedure of distance calculation to determine the suspected persons with COVID-19 via the infected person (the data of all mobile phone users including the distance of each of mobile will be sent to the central office).

2- Phase Two (authorized clinical center ):

Generally, the infected country with COVID-19 has assigned a number of authorized clinical centers in each area; the suspected persons can visit those centers to take COVID-19 test, if the test showed positive results, this person would be indicated as an infected person. At this stage, according to this framework the authorized clinical center should send the Id of infected person to the related telecom company to determine the people who were around this infected person in the last 14 days. This period of days has been proposed based on WHO official report. COVID-19 has an brooding period that shifts from 2 days to 14 days and is characterized initially by flu-like side effects but may lead into the creation of serious hacks, shortness of breath, stomach pain, loose bowels, sore throat, misfortune of taste and scent, tiredness, muscle aches/pain, rehashed shaking with chills, and fever[1].

\section{3- Phase Three (Telecom Company)}

At this stage of the framework, once the Id of infected person is known by the authorized clinical center, the responsible department such as RF (Radio frequency) department or other related field of the Telecom Company should search for mobile data of target person and collect information about the people who were around this person in the last 14 days and inform those people via sending SMS or making a phone call to visit the authorized clinical center to take COVID-19 test. At this stage, those people should be considered as suspected persons. 


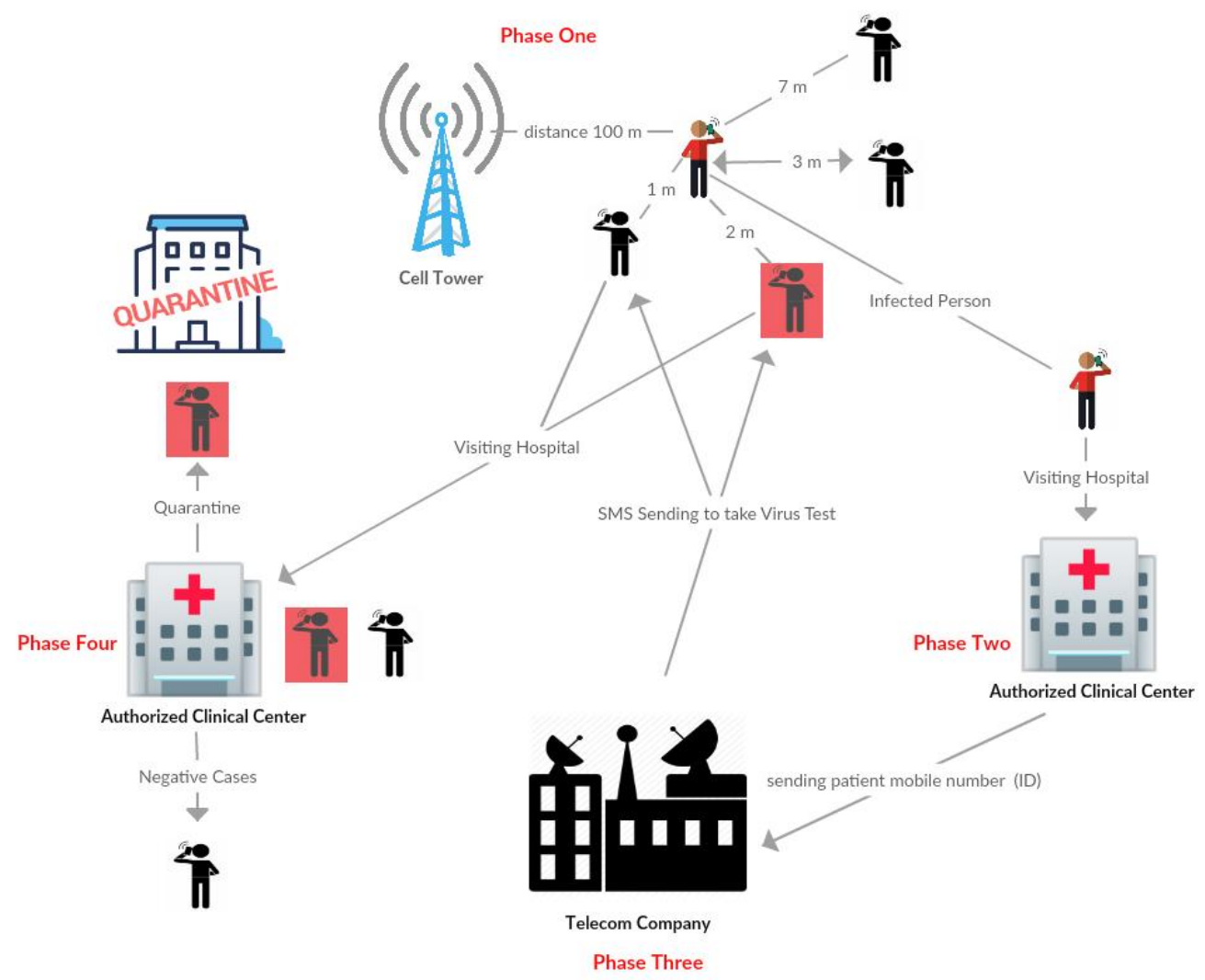

Figure 1: Proposed Framework to Control Covid-19

4- Phase Four (Evaluation and Dissection)

The last phase of the framework has been assigned as the final stage, in which the people who have been informed by the telecom company should visit the authorized clinical center. There they will be assigned to take the COVID-19 test and then persons with positive results will be sent to a quarantine to get the required treatments, while those with negative results test can follow his/her daily life style.

\section{CONCLUSION AND FUTURE WORK}

This paper is proposed of an applicable framework to be used as a tool to control the spread of coronavirus (COVID-19) by utilizing Cellular System. This framework comprised of two main parts. The first part defined and implemented a suitable formula to calculate the distance between mobile phones while switched on in which the Haversine formula was used to gain the sufficient outcome. Through the implementation of the so-called formula the calculation of the distances between the mobile phones with cell towers were made easier to be diagnosed. In the second part, an applicable framework was designed and through its implementation the suspected persons can be recognized. In addition, this can be regarded as a way to diagnose those who are unknown and were in touch with the person who is infected with COVID-19. The limitations of this paper might be defined as the occurrence of the amount of error in calculating the distances between points. And with increasing the number of mobile phone users in a day, the issue of analyzing and releasing the results are difficult tasks. It is suggested that, to make use of data mining to reduce the level of the occurrence of error in distance calculation. To address the issue of analyzing, it is suggested to use the big data technique which is implemented nowadays in telecom companies to deal with a huge data. 


\section{REFERENCE}

[1] J. Turner-Musa, O. Ajayi, and L. Kemp, "Examining Social Determinants of Health, Stigma, and COVID-19 Disparities," Healthcare, vol. 8, no. 2, p. 168, Jun. 2020, doi: 10.3390/healthcare8020168.

[2] World Health Organization. WHO timeline-COVID-19. Available online: https://www.who.int/newsroom/detail/27-04-2020-who-timeline---COVID-19 (accessed on 10 June 2020).

[3] http://www.emro.who.int/media/news/statement-by-who-regional-office-for-the-eastern- mediterranean-onCOVID-19-in-yemen.html (accessed on 12 June 2020).

[4] World Health Organization, "Coronavirus disease (COVID-19) situation reports", 2020 (accessed on 25 April 2020).

[5] S. Q. Salih, "Modified AlexNet Convolution Neural Network For COVID-19 Detection Using Chest X-ray Images," no. June, 2020, doi: 10.24017/COVID.14

[6] Q. Pham, D. C. Nguyen, T. Huynh-the, W. Hwang, and P. N. Pathirana, "Artificial Intelligence ( AI ) and Big Data for Coronavirus ( COVID-19 ) Pandemic: A Survey on the State-of-the-Arts," IEEE Trans. Artif. Intel., vol. PP, no. April, pp. 1-17, 2020, doi: 10.20944/preprints202004.0383.v1.

[7] P. D. Premlal and N. V. Eldhose, "Mobile tower radiation-an assessment of radiation level and its health implications in the residential areas of western ghats in idukki, Kerala," Int. J. Appl. Eng. Res., vol. 12, no. 20, pp. 9548-9554, 2017.

[8] S. Munjal, "CELL TOWER / BASE STATION ELECTROMAGNETIC RADIATION : LIVING ORGANISM , HEALTH CONSEQUENCES AND PROTECTION,” no. June, 2016.

[9] H. Wang, S. Xie, K. Li, and M. Omair Ahmad, "Big data-driven cellular information detection and coverage identification," Sensors (Switzerland), vol. 19, no. 4, pp. 1-23, 2019, doi: 10.3390/s19040937.

[10] M. H. A. Meniem, A. M. Hamad, and E. Shaaban, "Fast and Accurate Practical Positioning Method using Enhanced-Lateration Technique and Adaptive Propagation Model in GSM Mode," Int. J. Comput. Sci. Issues, vol. 9, no. 2, pp. 188-193, 2012.

[11] I. Vin, D. P. Gaillot, P. Laly, M. Liénard, and P. Degauque, "Overview of mobile localization techniques and performances of a novel fingerprinting-based method," Comptes Rendus Phys., vol. 16, no. 9, pp. 862-873, 2015, doi: 10.1016/j.crhy.2015.10.006.

[12] L. Cheng, C. Wu, Y. Zhang, H. Wu, M. Li, and C. Maple, "A survey of localization in wireless sensor network,” Int. J. Distrib. Sens. Networks, vol. 2012, 2012, doi: 10.1155/2012/962523.

[13] D. A. Prasetya, P. T. Nguyen, R. Faizullin, I. Iswanto, and E. F. Armay, "Resolving the shortest path problem using the haversine algorithm," J. Crit. Rev., vol. 7, no. 1, pp. 62-64, 2020, doi: 10.22159/jcr.07.01.11.

[14] R. Purbaningtyas, A. Arizal, and M. Sholehuddin, "Regional Leading Potential Recommendations: Implementation of Haversine Formula in Sidoarjo on Hands Mobile Applications," Probl. Inf. Technol., vol. 10, no. 2, pp. 70-76, 2019, doi: 10.25045/jpit.v10.i2.10.

[15] C. Nurul. Alam, K Manaf, A. R. Atmadja, and D. K. Aurum, "Implementation of Haversine Formula for Counting Event Visitor in The Radius Based on Android Application, ” 